\section{Imaging Adult Hippocampal Neurogenesis In Vivo}

Fifty years ago it was discovered that the dentate gyrus (DG) subfield of the hippocampus is one of the few areas of the brain where new neurons are generated throughout adulthood (Altman, 1963). In more recent years, advances in labeling techniques have allowed for a deeper understanding of how these adult-born granule cells (abGCs) mature and functionally integrate into the adult DG circuit. In humans, it was found that the majority of the DG GCs are subject to exchange, with about $\sim 700$ new neurons added to the hippocampus per day (Spalding et al, 2013). These nascent neurons have been implicated in a number of cognitive and mood-related functions, including some behavioral responses to stress and antidepressant treatments, as well as in pattern separation, the segregation of similar inputs into distinct output streams to facilitate memory encoding and discrimination (Kheirbek et al, 2012). However, these roles for abGCs in behavior and local circuit function have been primarily inferred from in vitro slice preparations, which lack the full complement of active inputs, or in vivo experiments where the intrinsic properties or total numbers of abGCs have been manipulated. This has left many questions unanswered, namely, how abGCs function and develop in vivo, and whether they encode information differently from mature GCs (mGCs).

To address these questions, recent studies have applied high-resolution imaging techniques allowing for the first direct observation of abGCs and mGCs in anesthetized and behaving animals. Danielson et al, (2016) used 2photon calcium imaging in head-fixed behaving mice to simultaneously measure activity patterns in mGCs and abGCs as mice explored a virtual environment (Figure 1). A tamoxifeninducible Nestin-CreER ${ }^{\mathrm{T} 2} /$ tdTomato mouse line was injected with a virus, inducing expression of GCaMP6f in all GCs, and pulsed with TMX 6 weeks before imaging to selectively label abGCs 6 weeks and younger with tdTomato. This revealed that abGCs were more active and less spatially tuned than their mature counterparts. In addition, unlike in mGCs, the most active abGCs tended to be the least spatially tuned, consistent with a model in which young abGCs are initially more active, and less spatially tuned, but within 6 weeks become more similar to the less active and more spatially tuned mature cells. Further elucidation of the developmental trajectory of abGCs in vivo has been provided by Goncalves et al, 2016, where abGCs were identified via delivery of a GFPexpressing retrovirus. Longitudinal visualization of dendritic structure by 2 photon microscopy revealed that abGC dendrites underwent overgrowth, followed by pruning as they integrated into the DG circuit, a process that was accelerated by enrichment and exercise. Future studies using these kinds of approaches will begin to elucidate how the unique functional and developmental properties of abGCs facilitate local circuit function and influence hippocampal output to impact behavior in both the mood and cognitive domains.

The development of novel approaches to visualize and manipulate discrete neuronal elements in vivo is paving the way to a more sophisticated, cell-type-based understanding of circuits that are disrupted in neuropsychiatric illness (Steinberg et al, 2015). As preclinical models indicate adult neurogenesis may have a role in emotional behavior, understanding the mechanisms by which abGCs function in vivo, and how their properties are impacted by environmental manipulations will clarify not only the normal encoding properties of these neurons but provide insight into how they may be targeted for therapeutic interventions.

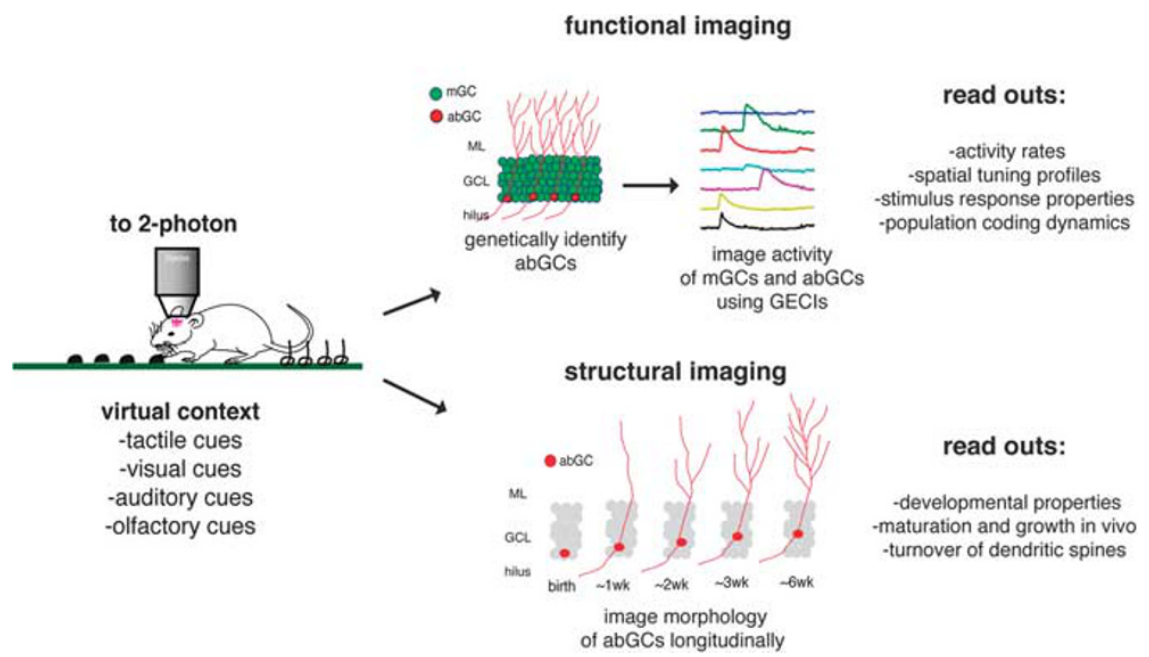

Figure 1. Observing abCG dynamics in vivo. In head-fixed mice, virtual contexts are generated via distinct combinations of diverse sensory stimuli. Genetically encoded calcium indicators (GECls) allow for 2-photon imaging of in vivo activity dynamics of mGCs and abGCs, while retroviral labeling allows for longitudinal analysis of abGC developmental dynamics. Such strategies will accelerate the understanding of how this form of plasticity contributes to hippocampal function and behavior. 


\section{FUNDING AND DISCLOSURE}

The authors declare no conflict of interest.

\section{ACKNOWLEDGMENTS}

MAK is supported by grants from NIMH (R01MH108623 and K01MH099371).

\section{Jeremy S Biane ${ }^{1}$ and}

Mazen A Kheirbek ${ }^{1,2,3}$

${ }^{1}$ Department of Psychiatry and Center for Integrative Neuroscience, University of California, San Francisco, California, USA; ${ }^{2}$ Kavli Institute for Fundamental Neuroscience, University of California, San Francisco, California, USA; ${ }^{3}$ Weill Institute for Neurosciences, University of California, San Francisco, California, USA

E-mail: Mazen.Kheirbek@ucsf.edu

Altman $J(1963)$. Autoradiographic investigation of cell proliferation in the brains of rats and cats. Anat Rec 145: 573-591.

Danielson NB, Kaifosh P, Zaremba JD, Lovett-Barron M, Tsai J, Denny CA et al (2016). Distinct contribution of adult-born hippocampal granule cells to context encoding. Neuron 90 101-112.

Goncalves JT, Bloyd CW, Shtrahman M, Johnston ST, Schafer ST, Parylak SL et al (2016). In vivo imaging of dendritic pruning in dentate granule cells. Nat Neurosci 19: 788-791.

Kheirbek MA, Klemenhagen KC, Sahay A, Hen R (2012). Neurogenesis and generalization: a new approach to stratify and treat anxiety disorders. Nat Neurosci 15: 1613-1620.

Spalding KL, Bergmann O, Alkass K, Bernard S, Salehpour M, Huttner HB et al (2013). Dynamics of hippocampal neurogenesis in adult humans. Cell 153: 1219-1227.

Steinberg EE, Christoffel DJ, Deisseroth K, Malenka RC (2015). Illuminating circuitry relevant to psychiatric disorders with optogenetics. Curr Opin Neurobiol 30: 9-16.

Neuropsychopharmacology Reviews (2017) 42, 373-374; do: I 0. I 038/npp.2016.200

\section{Emerging Epigenetic Therapies in Neuroscience: Focus on Bromodomain- Containing Drug Targets}

Failure to appropriately control gene expression is a salient feature of most human diseases. Therefore, the field of epigenetic therapeutics has rapidly grown into one of the most active areas of drug discovery. This is fueled by the fact that there exist hundreds of socalled druggable (by small molecules) epigenetic targets, many of which affect the state of chromatin and therefore gene expression. The result of this type of drug action, exemplified here by bromodomain inhibitors, is typically an alteration of the expression of a number of genes (up- as well as down-regulation). While some genes are more susceptible to this type of regulation, there is marked context dependency that represents both a challenge and an opportunity to drug hunters.

The bromodomain module, a critical component of epigenetic regulation, selectively recognizes acetylated lysine residues present in both histone and non-histone proteins. In human cells, there exist 46 proteins that contain bromodomain(s). The dysregulation of these so-called protein reader functions, and the genes that they control downstream, have been implicated in the development of a variety of diseases, making them attractive targets for drug discovery. It has been known for about a decade that small molecules are capable of binding to a bromodomain and the number of reported inhibitors has expanded dramatically in the past few years. The profound and broad pharmacology of bromodomain inhibition, especially that associated with targeting the so-called BET subfamily of bromodomains (BRD2, BRD3, BRD4, and BRDT), has led to the progression of a number of small molecules into the clinic for liquid as well as solid tumors. However, these BET bromodomain inhibitors may also have utility for non-malignant diseases of the nervous system.

BET bromodomain inhibitors can influence the differentiation and maturation of a variety of cell types. Indeed, anti-cancer effects, mediated primarily by modulation of BRD4, have been the main driver of drug discovery thus far. For example, strong inhibitor efficacy can be observed in models of glioblastoma, which has stimulated the discovery of novel ligands with high brain exposure (Pastori et al, 2015). Moreover, much interest has been placed on immunomodulatory activi- ties including altered expression of a number of cytokines. Indeed, BET bromodomain inhibitors hold promise to be used for the treatment of brain disorders characterized by neuroinflammation, including Alzheimer's disease (Magistri et al, 2016).

Like other epigenetic modulators, BET bromodomains could conceivably be employed to correct single gene disorders. An example thereof is the hexanucleotide repeat expansion residing within the C9ORF72 gene, representing the most common known cause of amyotrophic lateral sclerosis (ALS). Indeed, BET bromodomain inhibitors increase the expression of C9ORF72 mRNA and pre-mRNA and may therefore compensate for haploinsufficiency without increasing the production of toxic RNA and protein products (Zeier et al, 2015).

Moreover, epigenetic phenomena have often implicated in memory as well as addiction. Korb et al, 2015 demonstrated that BRD4 provides a critical link between neuronal activation and the transcriptional responses that occur during memory formation.

In recent studies, we observed that BRD4 is elevated in the nucleus accumbens and recruited to promoter regions of addiction-related genes following repeated cocaine administration, and that inhibition of BRD4 attenuates transcriptional and behavioral responses to cocaine (Sartor et al, 2015). Thus, it is possible that bromodomain inhibitors may have therapeutic utility in the treatment of cocaine and perhaps other addictions.

Importantly, it must be noted that epigenetic drug will affect the expression of a number of genes and that the undesired effects can arise as a consequence (eg, Sullivan et al, 2015). However, it is reasonable to assume that the most critical period for putative adverse effects would occur early in development.

In conclusion, a variety of epigenetic drug candidates-mostly thanks to efforts in the cancer field-have recently become available to the field of neuroscience. This offers a tremendous opportunity that must be seized. This brief piece has focused on BET 